\title{
Vectorial Channel Estimation for Uplink MC-CDMA in Beyond 3G Wireless Systems
}

\author{
P. Marques ${ }^{1,2}$, A. Gameiro' and J. Fernandes' \\ ${ }^{1}$ Universidade de Aveiro, Instituto de Telecomunicações, DETUA, 3810 Aveiro, Portugal \\ ${ }^{2}$ Escola Superior de Tecnologia - Instituto Politécnico de Castelo Branco, 6000 Castelo Branco, Portugal \\ emails: pmarques@av.it.pt, amg@det.ua.pt, zf@det.ua.pt
}

\begin{abstract}
In beyond $3 G$ wireless systems the bandwidth efficiency can be increased with the use of adaptive antenna arrays. This paper focus on a key issue for adaptive antenna arrays, that is, channel parameters estimation including Direction-Of-Arrival (DOA). In order to estimate DOA, the channel frequency responses for the links between the mobile users and each of base station array elements are estimated by pilot-aided minimum mean square error (MMSE) algorithm. This estimator is not sensitive to the channel statistics. Based on frequency response estimatives DOA's of impinging multipath components to the base station are estimated by a low complex Maximum Likelihood (ML) approach. Furthermore an uplink burst structure with specifically designed midamble field for multiuser channel estimation in MC-CDMA is proposed. The performance is assessed in terms of channel estimation errors for a MC-CDMA TDD system over fast and slow fading mobile channels.
\end{abstract}

Keywords- beyond 3G; MC-CDMA; channel estimation; direction of arrival; multipath

\section{INTRODUCTION}

Multi-carrier code division multiple access (MC-CDMA) [1] is one of the promising techniques for achieving the high-speed data required in Beyond $3 \mathrm{G}$ mobile communication systems. A very important feature of a MC.CDMA system is the capacity to spread the signal bandwidth without increasing the adverse effect of inter symbol interference (ISI). In contrast to DS-CDMA, used on UMTS system, MC-CDMA does not multiply the user signal by a chip sequence, but the sarve user bit is transmitted on multiple subcarriers simultaneously. If the bandwidth of each subcarrier is much less than the channel coherence bandwidth, a frequency flat channel model can be assumed for each subcarrier. Moreover, inserting a cyclic prefix results in an ISI free channel assuming that the length of the guard interval is greater than the delay spread of the channel. Therefore, the effect of the multipath channel on each subcarrier can be represented by a single complex multiplier, affecting the amplitude and phase of each subcarrier. Hence, the equalizer at the receiver can be implemented by a set of complex multipliers, one for each subcarrier. Since the channel information is required for the equalization operations, channel estimation plays an important role in MC-CDMA system design. The transmitter uses certain tones, called pilot tones, multiplexed into the transmitted data stream. The channel frequency response can be estimated using the MMSE criterion and interpolation between pilot tones [2].

In MC-CDMA systems capacity is limited by the Multiple Access interference (MAI) occurring when several mobile terminals share the mobile radio channel. Beamforming and Space-Division Multiple Access (SDMA) using multiple antennas are considered as effective means to combat MAI by separating the users signals in space [3][4]. Hence, users having sufficiently different spatial signatures can share the same spreading code increasing the system capacity without a significant impact on MAI. Moreover, an interesting feature of the Time Division Duplex (TDD) mode is the ability, according the mobile station (MS) velocity, to benefit from the channel reciprocity between uplink and downlink. Indeed, we can consider that the spatial signatures remains almost unchanged between consecutive uplink and downlink transmission time slots. Therefore, it is possible to use the uplink channel estimates to perform transmit beamforming allowing the use of low complex algorithms at the MS even in the presence of high MAI [5]. The spatial signature of a userr is determined by the Direction-Of-Arrival (DOA) of the different paths forming the uplink multipath channel between the user and base station antenna array. In many approaches of adaptive antenna applications it is useful to guess DOA. Our goal is to design an estimation scheme to provide accurate estimates for the DOAs of the multipath components impinging on the base station antenna array. Based on channel frequency response estimated for each array element, using robust MMSE algorithm, the DOA's of impinging multipath components to the base station are estimated by a low complex Maximum Likelihood (ML) approach. Other objective of this paper is to investigate the effect of the pilot tones spacing and mobile speed on error of channel frequency response and DOA estimatives.

The paper is organized as follows. Section II presents system description. Section III presents the uplink burst structure for MC-CDMA / TDD system. Section IV proposes a robust MMSE pilot-aided channel estimation algorithm to estimate the channel frequency response. Section $\mathrm{V}$ we present an extension of the algorithm using the ML principle to estimate the DOA. Section VI gives some simulation results and conclusions are given in Section VII.

\section{SYSTEM DESCRIPTION}

In MC-CDMA systems, the original data symbol $d_{k, p}$ with duration $(T d)$ is increased by splitting the high bit rate stream into many low-rate parallel streams. In order to eliminate interference between parallel data streams, each low-rate data stream modulates orthogonal subcarriers by means of the IFFT operation. The same basic structure is used in both Orthogonal Frequency Division Multiplex (OFDM) and MC-CDMA. The difference is that in OFDM an individual data symbol is carried on a single subcarrier, whereas in MC-CDMA each individual data symbol is spread across a set of $S F$ subcarriers, where $S F$ is the length of the frequency domain spreading code $c_{k}$, that we assume to be WalshHadamard. The transmitted signal can be written as: 


$$
x_{k}(t)=\sum_{p=0}^{\frac{N c}{W}} \sum_{n=0}^{1} d_{k, p}(t) c_{k, n}(t) e^{\left.i 2 \pi\left(f_{n}+(p)+1 \%+n\right) \Delta f\right) t}
$$

where $N_{c}$ is the total number of subcarriers; $f_{i}$ is the first RF carrier; $\Delta f=1 / T S$ is the subcarrier spacing and $T S$ is the duration of a whole MC-CDMA symbol, that is $T s=T d N C / S F+C P$. The cyclic prefix $(C P)$ is added to eliminate the effect of the ISI. The received signal at the base station is a superposition of multiple copies of attenuated and delayed signals transmitted by all the $K$ users. The radio channel of each user in multipath environment can be modelled by a tap-delay-line,

$$
h_{k, m}(t)=\sum_{l=l}^{l} \alpha_{k, l} e^{i \Omega_{k, l}} \delta\left(t-\tau_{k, l}\right)
$$

where:

$$
\begin{aligned}
& k:(1 \ldots K) \text { refers to the } k t h \text { user } \\
& m:(0 \ldots M-l) \text { refers to the } m t h \text { array element }
\end{aligned}
$$

l:(1...L) refers to the th channel path

$\alpha_{k . l}$ is the amplitude of the 1 th multipath component of user $k$ wich is considered identical for the all $M$ elements

$\Omega_{k . l}=\theta_{k . l}+a_{m}\left(D O A_{k . l}\right)$ where $\theta_{k, l}$ is the phase of the Ith multipath component of user $k$ and $a_{m}\left(D O A_{k, l}\right)$ is the phase shift of the array steering vector for the direction $D O A_{k, 1}$.

$\tau_{k, l}$ is the path $/$ th delay of user $k$;

Therefore, the signal at the $m$ th sensor is given by,

$$
r_{m}(t)=\sum_{k=1}^{K} \sum_{l=1}^{l} \alpha_{k, l} e^{j / \Omega_{k, l}} x_{k}\left(t-\tau_{k, l}\right)+n_{m}(t)
$$

The additive noise processes present at each antenna, $n_{m}$, are assumed to be uncorrelated white noise complex Gaussian with zero mean and power spectral density $\sigma_{n}^{2}$. In this system model $n_{m}$ also include the intercell interference due to other cells. The $M$ signals received at each antenna array element are processed by vectorial channel estimator before the space-time combing technique.

\section{UPLINK BURST STRUCTURE}

In contrast to the downlink, where the signal received by a MS passed through the same channel, in uplink the received signal is the sum of $K$ different signals filtered by $K$ different channels. This makes the channel estimation operation much more difficult because it is necessary to estimate $K$ different channels. In this communication the proposed channel estimator is pilot-symbol assisted, that is, $K$ known vectors $\left(X p_{k}\right)$ are multiplexed in frequency domain composing one midamble field in burst structure.

\section{A. Selection of pilot pattern}

The most important parameters for the selection of a pilot pattern are the expected maximum speed, which determines he minimum coherence time, and the maximum excess delay, which determines the minimum coherence bandwidth. The jilot pattern design problem is a tradeoff between good channel estimation (closely spaced pilots) and high spectral and power efficiency (sparsely spaced pilots). As discussed in [6], the grid density of the pilot symbols must satisfy the 2-D sampling theorem in order to recover channel parameters, that is,

$$
f_{d \max } T s D_{1} \leq 1 / 2 \text { and } \tau_{\max } \Delta f D_{f} \leq 1 / 2
$$

where $D_{l}$ denote the spacing in terms of MC-CDMA symbols between two pilot subcarriers in the time domain and $D_{f}$ is used to denote the interval in terms of the number of subcarriers between two adjacent pilots in the frequency domain. It is suggested in [6] that an oversampling rate of two for the pilot symbols be used to improve the estimation performance. In (4) $f_{d \max }=f_{c} v_{\max } / c$ is the maximum Doppler frequency where $c$ is the speed of light, $v_{\max }$ is the maximum mobile speed and $f_{c}$ is the carrier frequency. $\tau_{\text {max }}$ represents the maximum path delay present in power delay profile of radio channel. For the simplicity and robustness of a channel estimator an adjustment according to the worst-case scenario for the Doppler frequency and excess delay is often suggested, i.e. to minimum correlation in the time and frequency.

We consider the parameters defined for $4 \mathrm{G}$ systems within the MATRICE project [7], $f_{\mathrm{c}}=5 \mathrm{GHz}, v_{\max }=300 \mathrm{Km} / \mathrm{h}$, (resulting in a maximum Doppler shift $f_{\text {ctmax }}=1.38 \mathrm{KHz}$ ) and RF bandwidth of $\mathrm{W}=57.6 \mathrm{MHz}$. The number of subcarriers was considered to be $N_{c}=1024$. The time between symbol samples $\Delta=17.4 \mathrm{~ns}$. Considering that the cyclic prefix has $20 \%$ of $\mathrm{MC}$ CDMA symbol duration, implies $T_{S}=1.2 N c \Delta=21.3 \mu \mathrm{s}$. With these parameters and assuming an oversampling factor of two, the pilot distance in time must comply with $T_{s} D_{t}<181 \mu \mathrm{s}$, or $D, \leq 8$ MC-CDMA symbols. For the frequency separation, considering the worst-case scenario of the BRAN C outdoor radio channel with maximum delay of $1050 \mathrm{~ns}$ [8], an oversampling factor of two implies that the pilot distance in must comply with $\Delta f D_{f}<238 \mathrm{KHz}$, or $D_{f} \leqslant 4$ subcarriers.

\section{B. Uplink TDD burst structure}

The uplink data structure is shown in Fig. 1, where two MC-CDMA symbols from known pilot tones (midambles) are included between the data symbols. For each user the pilots tones are multiplexed in the frequency domain with the pilots of others users, thus the channel estimation is not sensitive to the multiple access interference. In this structure, and in order to allow proper operation of the pilot-aided channel estimator, the burst forms a 15 MC-CDMA symbol set with duration 345 $\mu$ s. It includes also a guard time interval of $25 \mu$ s between TDD bursts, equal to the one used in UMTS-TDD system. The midamble overhead achieved with this burst structure is $12 \%$, lower that the $20 \%$ of UMTS-TDD burst type 1 . Notice that the maximum number of different channels estimated by this technique is $K=2 D_{f}$. 


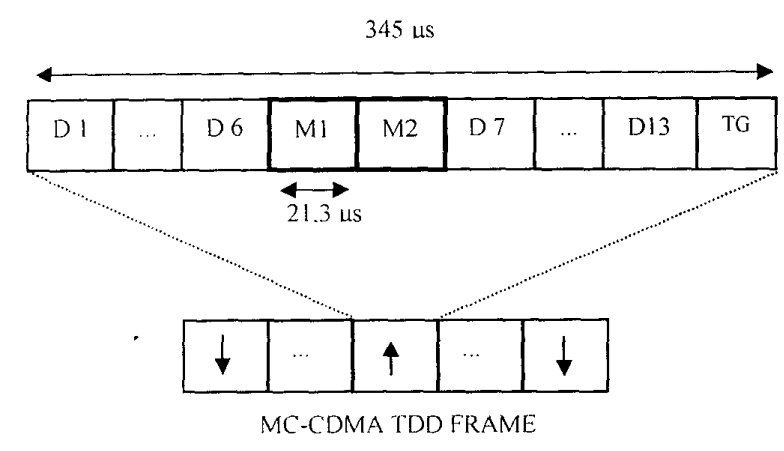

Fig. I MC-CDMA uplink burst structure and possible TDD system frame.

\section{PILOT-Aided ChanNel FREQUeñCy Response ESTIMATION}

The channel frequency response estimation algorithm performs two sequential operations: first the pilot symbols are estimated, and afterwards an interpolation operation is performed to estimate the frequency channel response at other subcarriers. The pilot signal estimation based on a MMSE criterion, together with channel polynomial interpolation is presented hereafter.

\section{Robust MMSE frequency response estimation in pilot locations}

To reduce the computational complexity the pilot signals $X p$ are complex symbols with equal values. The length of $X p$ is $P=N c / D_{f}$. Let

$H p=[H(0) H(1) \ldots H(P-1)]^{\mathrm{T}}$

be the channel response at the pilot subcarriers, and

$Y p=[Y p(0) Y p(1) \ldots Y p(P-1)]^{\mathrm{T}}$

be the vector of received pilot signals.

The received pilot signal vector $Y p$ can be expressed as

$Y_{p}=X p H p+1 p+n p$

where $X p$ is a $P$-by- $P$ diagonal matrix of pilots,

$X p=\operatorname{diag}(x p(0), \ldots, x p(P-1))$.

$I p$ is the vector of intercarrier interference and $n p$ is the vector of i.i.d Gaussian noise with variance $\sigma_{n}^{2}$ assumed to be known. In the conventional Least Squares (LS) channel estimation method the pilot signals estimates are obtained by a simple division by the known pilot values,

$$
\hat{H}_{p t s:}=X p^{-1} Y p
$$

which makes the LS estimate of $H p$ very susceptible to Gaussian noise and inter-carrier interference. Furthermore, because the channel responses of data subcarriers are obtained by interpolating the channel responses of pilot subcarriers, the performance of channel estimator is highly dependent on the accuracy of the of pilot signals estimates. Significantly better results are obtained with the MMSE method which exploits the channel correlation in the frequency domain. The mathematical representation for MMSE estimator of pilot signals is [9],
$\hat{H}_{p M M S E}=R_{f p}\left(R_{f p}+\sigma_{n}^{2} I\right)^{-1} \hat{H}_{p l S}$

where $I$ is a $P$-by- $P$ identity matrix and $R_{f p}$ is the frequency-domain correlation matrix. The major drawback of the MMSE pilot estimation is the requirement of the knowledge of $R_{f p}$, which is not very realistic since this matrix is time-varying and changes with the environment and terminal speed. The key idea we used to solve this problem is to perform a first estimate of the pilots $\left(\hat{H}_{p l . S}\right)$ using the LS criteria, and compute an estimate for $R_{f p}$ based on these estimates [2],

$$
R_{f p} \approx E\left[\hat{H}_{p l s}, \hat{H}_{p L S}^{H}\right\rfloor
$$

where $^{H}$ denotes conjugate transpose.

The matrix estimate obtained through (10) is then used in (9). This approach is robust in the sense that it can cope with different channel statistics.

\section{B. Channel interpolation in fréquency domain}

Interpolation in the time domain originates latency due to pilot symbol buffering in TDD services operation. Thus we only consider an interpolation operation in the frequency direction on MC-CDMA subcarriers. We consider the second-order polynomial interpolation because this leads to a moderate computational complexity. In the second-order interpolation algorithm, three successive pilot subcarriers are used to determine the channel response for data subcarriers that are located in between the pilots.

\section{MaXimUM LIKELIHOOD DOA ESTIMATION}

As shown in Fig. 2, the signal $x_{k}(t)$ is transmitted through a frequency selective multi-path fading channel with impulsc response $h_{k, m}$. For each array element, the channel estimatoi removes the cyclic prefix of MC-CDMA symbol in the signal $r_{m}(t)$ and the resulting signal samples undergo an FFT operation thai demultiplexes the multicarrier signals. After that, the received pilosignals $Y p_{k}$ are extracted from FFT output and the robus MMSEchannel estimation algorithm estimates the frequency channel response for each antenna array $\left(\hat{H}_{k, m}\right)$. Following the $\hat{L}$ frequency-domain estimates are transformed into $M$ time-domai impulse response by the IFFT operation, leading to,

$\hat{h}_{k, m}=\operatorname{IFFT}\left\{\hat{H}_{k, m}\right\}=\left\lfloor\hat{h}_{k, m}(1), \ldots, \hat{h}_{k, m}\left(N_{c}\right)\right\rfloor$

After a time domain representation of the channel is obtained, : "Path search" procedure detects the $L$ most important multipati components of $\hat{h}_{k, m}$, and then the DOA for each path $\mathrm{i}$ independently estimated using the ML criterion. Notice that th time resolution between successive multipaths is equal to $\Delta=1 / \mathrm{W}$ In case of radio channel with $L$ multipath components an considering $K$ users, the number of paths needed to be resolved ca be quite large $(K x L)$. Straightforward application of ML estimatic principles implies that log-likelihood function must be maximize with respect to the unknown parameters (amplitude, phase an DOA), for each multipath component. This results in a non-linea $K \times L \times 3$-dimensional, maximization problem, which i computationally very intensive. 


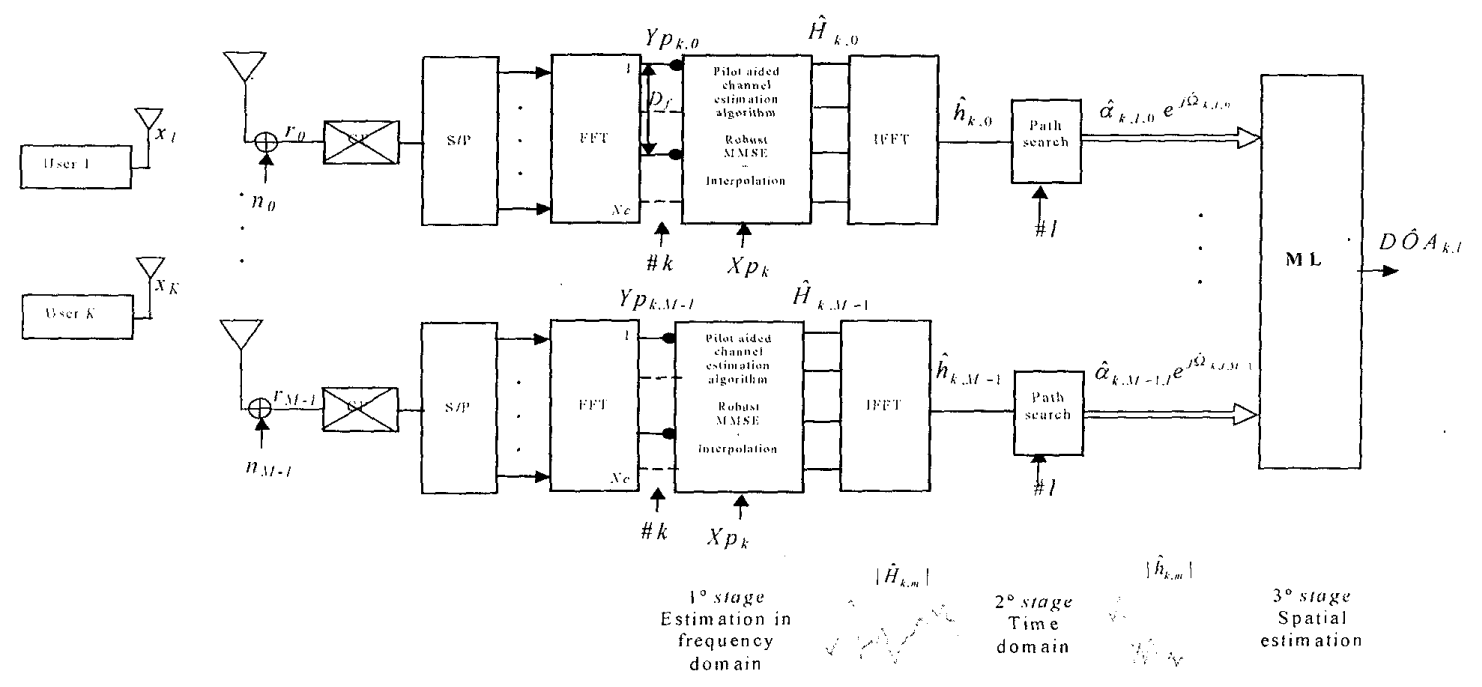

Fig.2 ML DOA estimation unit based in $M$ robust MMSE pilot-aided channel estimator.

To overcone this limitation, we implemented a moderate complexity ML DOA estimation based on the robust MMSE frequency channel estimator. The basic structure shown in Figure 2: we apply the robust MMSE to each of the $M$ array elements, and then estimate the DOA using the ML criterion based on the phase shift relation existing between array elements. This algorithm is of course no longer the optimum joint parameter estimator but it brings down the $K \times L \times 3$-dimensional maximization problem to $K \times L$ successive 3-dimensional problems, thus reducing the computational complexity. The mathematical formulation of $\mathrm{ML}$ criterion for DOA estimation, using a Uniform Circular Array (UCA), is detailed in [10], and after some mathematical manipulation it can be written as,

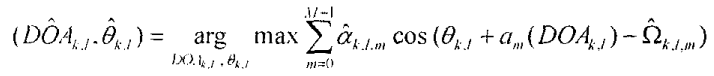

This technique does not make any assumption about the angular separation between the DOAs of the different paths. However the performance of the ML DOA estimation technique depends heavily on the quality of the scalar channel estimation provided by robust MMSE as we will see in the next Section.

\section{Simulation Results}

To evaluate the performance of the proposed channel stimation algorithm (robust MMSE), the mean square error MSE) was analysed for BRAN $\mathrm{C}$ channel model with iifferent mobile terminal speeds, and pilot subcarries :eparations $\left(D_{f}\right)$. The MSE is defined as

$$
M S E=\frac{1}{N s N c} \sum_{s=1}^{N s} \sum_{n=1}^{N c}\left|H_{n, s}-\hat{H}_{n, s}\right|^{2}
$$

where $N S$ is the number of MC-CDMA symbols simulated. The MC-CDMA burst structure used in the simulations is the burst presented in Section II. Table $I$ shows the parameters used in the MC-CDMA simulation chain.

The simulation results in Fig. 3 show the effect of the pilot symbol spacing on the Mean Square Error (MSE) obtained with robust MMSE estimator, where we can see that for $D_{F}=16$ the degradation is high.

TABLE 1: PARAMETERS OF THE SIMULATED MC-CDMA SYSTEM.

\begin{tabular}{|l|l|}
\hline$R F$ bandwidth & $57.6 \mathrm{MHz}$ \\
\hline Carrier frequency & $5 \mathrm{GHz}$ \\
\hline Number of FFT tones & 1024 \\
\hline Base station array & $\mathrm{UCA}$ \\
\hline Number of elements & 8 (spaced by $\lambda / 2$ ) \\
\hline Doppler spectrum & Classical Rayleigh fading \\
\hline
\end{tabular}

The simulation results in Fig. 4 show the influence of different mobile speed, or Doppler effect, on the MSE. When the mobile velocity is increased from $3 \mathrm{Km} / \mathrm{h}$ to $300 \mathrm{Km} / \mathrm{h}$, as shown in Fig. 4, the performance degradation of the estimator is moderate, notice that the proposed algorithm does not includes interpolation in time domain because of the latency times this would introduce in TDD services.

For DOA estimation performance analysis we extended BRAN C tap-delay model to a space-time model by allocating a DOA, seen from the BS antenna array, to each of these paths. DOAs are chosen randomly according to a uniform 
distribution that we assume to remain constant for the simulation. The DOA rms error for the strongest channel multipath component obtained with ML algorithm was simulated for different values of pilot separation and the results are shown in Fig. 5.

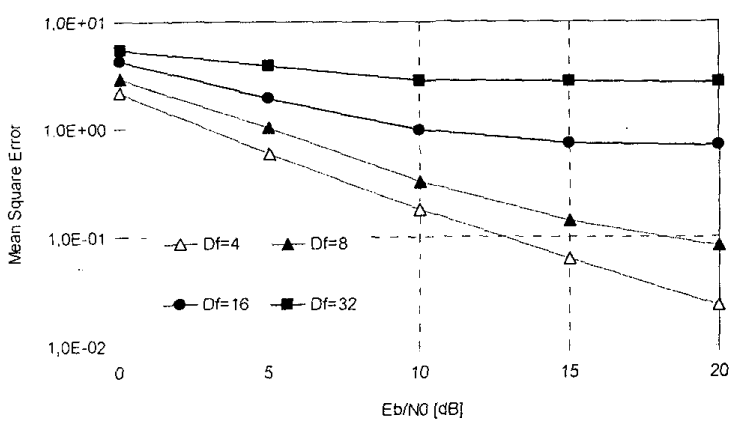

Fig. 3 MSE for robust MMSE channel frequency estimation algorithms with $D_{i}=4 . v=3 \mathrm{~K} \mathrm{~m} / \mathrm{h}$, channel model BRAN C.

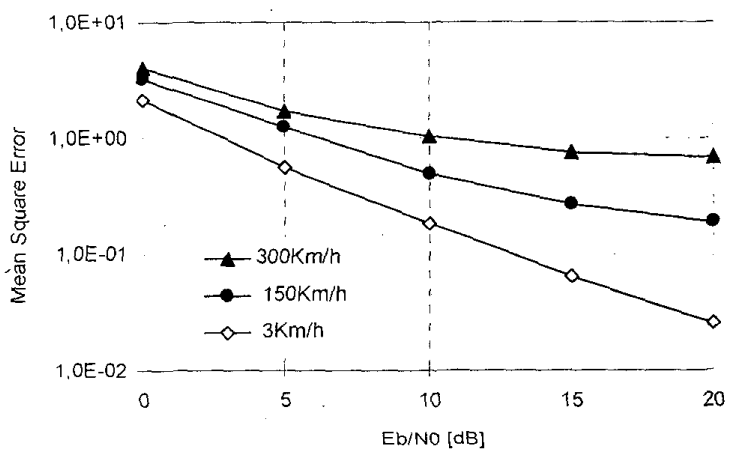

Fig. 4 Effect of mobile speed on MSE for robust MMSE algorithm with $D_{F}=4$, channel model BRAN C.

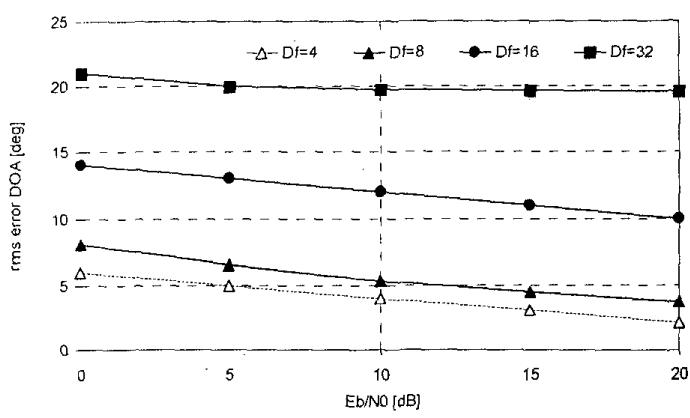

Fig. 5 Effect of pilot separation on error DOA using ML algorithm with $v=3$ $\mathrm{K} m / \mathrm{h}$, strongest path of channel model BRAN $\mathrm{C}$.

\section{CONCLUSIONS}

In this paper, a combined channel frequency estimation scheme for a TDD MC-CDMA mobile radio system and DOA estimation algorithm was presented. A robust MMSE channel estimation algorithm for the frequency channel statistic variation that does not rely on a priori knowledge of the channel statistics was proposed. The DOA estimation is performed resorting to a ML criterion. The complete algorithm has been tested in a link level simulation platform for BRAN $C$ channel model and uniform circular array. Simulations have shown that the DOA estimation algorithm using $D_{f}=4$ and $\nu=3 \mathrm{Km} / \mathrm{h}$ guarantees a DOA rms error below $6^{\circ}$, from low to high values of Eb/No. In these conditions the maximum number of users per time slot allowed by uplink burst structure is 8 . If the number of users increases then the pilot tones separation and the rms error of DOA increases too.

\section{ACKNOWLEDGMENT}

The work presented in this paper was supported by the European IST project MATRICE (MC-CDMA Transmission Techniques for Integrated Broadband Cellular Systems).

\section{REFERENCES}

[1] N. Yee, J. Linnartz, G. Fettwis,, "Multi-carrier CDMA in indoor wireless network", PIMRC, Set. 1993.

[2] P. Marques, A. Gameiro and J. Fernandes, "Pilot-Aided Channel Estimation for MC-CDMA Beyond 3G Wireless Systems". IST SUMMIT, Aveiro, Portugal, June 2003

[3] C. Kim, K. Lee and Y. Cho, "Adaptive Bearnforming Algorithm for OFDM Systems with Antenna Arrays". IEEE Trans. Consumer Electronics, vol. $46, N^{\circ} 4$, Nov. 2000.

[4] K. Wong, R. Cheng and R. Murch, "Adaptive Antennas at the Mobile and Base Stations in an OFDM/TDMA System", IEEE Trans. Commun. vol. $49, N^{\circ}$ I, Jan. 2001

[5] T. Salzer and D. Mottier, "Transmit Beamforming for SDMA in MultiCarrier CDMA Downlink on a Per Subcarrier Basis" IEEE Int. Cont. on Telecomnunication, June 2003.

[6] J. Moon, S. Choi, "Performance of channel estimation methods fo OFDM systems in a multipath fading channels", IEEE Trans. Consume: Electronics, vol. 46, No 1, Feb. 2000.

[7] F. Migneret, L. Girard, "4G Reference scenario specification: basic parameters and services", IST MATRICE Project, Deliverable DI.I Mar. 2002.

[8] ETSI Project Broadband Radio Access Networks (BRAN); HIPERLAI Type 2, Technical specitication; Physical layer, Oct. 1999.

[9] M. Chang, Y. Su, "Model-based channel estimation for OFDM signal: in Rayleigh fading ", JEEE Trans. Commun., vol. 50, No 4, April 2002

[10] P. Marques, A. Gameiro and I. Fernandes, "Vectorial multipath channel estimation for the UTRA-TDD mode", IEEE Vehicular Tech. Conf, Atlantic City, Oct. 2001. 